Maturitas Volume 100, 1 June 2017, Pages 49-56

\title{
Effect of exercise on sleep quality and insomnia in middle- aged women: A systematic review and meta-analysis of randomized controlled trials(Review)
}

- Rubio-Arias, J.Á. ${ }^{a}$,

- Marín-Cascales, E. a,

- Ramos-Campo, D.J.a,

- Hernandez, A.V.

- Pérez-López, F.R. ${ }^{\circ} E m a i l$ Author

- View Correspondence (jump link)

- $\quad{ }^{a}$ Department of Physical Activity and Sports Sciences, Faculty of Sports and Research Center for High Performance Sport, Catholic University of Murcia (UCAM), Murcia, Spain

- 'School of Medicine, Universidad Peruana de Ciencias Aplicadas (UPC), University of Connecticut/Hartford Hospital Evidence-based Practice Center, 80 Seymour St, Hartford, CT, United States

- 'Department of Obstetrics and Gynecology, University of Zaragoza Faculty of Medicine and Lozano-Blesa University Hospital, Domingo Miral s/n, Zaragoza, Spain

Abstract_View references (47)

Objective We assessed the effects of programmed exercise (PE) on sleep quality and insomnia in middle-aged women (MAW). Methods Searches were conducted in five databases from inception through December 15, 2016 for randomized controlled trials (RCTs) evaluating the effects of PE versus a non-exercising control condition on sleep quality, sleep disturbance and/or insomnia in MAW.

Interventions had to last at least 8 weeks. Sleep quality was assessed with the Pittsburgh Sleep Quality Index (PSQI) and insomnia with the Insomnia Severity Index (ISI). Random effects models were used for meta-analyses. The effects on outcomes were expressed as mean differences (MDs) and their $95 \%$ confidence intervals $(\mathrm{Cl})$. Results Five publications reported data from four RCTs on PE effects during $12-16$ weeks on sleep quality ( $n=4$ studies reporting PSQI results) and/or insomnia ( $\mathrm{n}=3$ studies reporting ISI results), including 660 MAW. Lowmoderate levels of exercise significantly lowered the PSQI score (MD $=-1.34$; $95 \% \mathrm{Cl}-2.67,0.00 ; \mathrm{p}=0.05)$ compared with controls. In a subgroup analysis, moderate PE (aerobic exercise) had a positive effect on sleep quality (PSQI score $\mathrm{MD}=-1.85 ; 95 \% \mathrm{Cl}-3.62,-0.07 ; \mathrm{p}=0.04$ ), while low levels of physical activity (yoga) did not have a significant effect (MD-0.46, 95\% Cl -1.79, 0.88, $\mathrm{p}=0.50$ ). In three studies (two studies of yoga, one study of aerobic exercise), there was a nonsignificant reduction in the severity of insomnia measured with the ISI score (MD $-1.44,95 \% \mathrm{Cl}-3.28,0.44, \mathrm{p}=0.13$ ) compared with controls. Heterogeneity of 
effects among studies was moderate to high. Conclusion In middle-aged women, programmed exercise improved sleep quality but had no significant effect on the severity of insomnia. (C) 2017 Elsevier B.V.

Author keywords

- Exercise

- Insomnia

- Insomnia severity index

- Middle-aged women

- Pittsburgh sleep quality index

- Yoga

Indexed keywords

EMTREE medical terms: aerobic exerciseexercisefemalehumaninsomniaInsomnia Severity Indexmeta anal Sleep Quality Indexrandomized controlled trial (topic)Reviewrisk assessmentslee qualitysystematic reviewyoga

- ISSN: 03785122

- CODEN: MATUD

- Source Type: Journal

- Original language: English

- DOI: 10.1016/j.maturitas.2017.04.003

- Document Type: Review

- Publisher: Elsevier Ireland Ltd 